\title{
UNIFORM ASYMPTOTIC EXPANSIONS OF THE MODIFIED \\ BESSEL FUNCTION OF THE THIRD KIND OF LARGE IMAGINARY ORDER ${ }^{1}$
}

\author{
BY CHARLES B. BALOGH
}

Communicated by A. Zygmund, August 9, 1965

The modified Bessel equation

$$
\frac{d^{2} w}{d z^{2}}+\frac{1}{z} \frac{d w}{d z}-\left(1-\frac{\nu^{2}}{z^{2}}\right) w=0
$$

with its particular solution $w=K_{i v}(z)$, the modified Bessel function of the third kind with pure imaginary order is of fundamental significance in the diffraction theory of pulses. Moreover this function is the kernel of the Lebedev transform [3].

With the exception of Friedlander's results [2] little information is available about the behavior of $K_{i \nu}(z)$ when both $\nu$ and $z$ are large which case is of great importance in the applications. In [2] Langer's differential equation method is applied and an asymptotic formula is given for the function and for the zeros of its derivative.

The aim of this paper is to give a fairly complete description of $K_{i \nu}(z)$ for $\nu \rightarrow \infty$; the proofs will be given elsewhere.

Based on (1) and on Olver's Theorem B [4], [6] a uniform asymptotic series is constructed in terms of the Airy function $A i(\xi)$ and of its derivative $A i^{\prime}(\xi)$ for $\nu \rightarrow \infty$ in a region $R$ which contains the sector $\operatorname{Re} z \geqq 0, z \neq 0$.

$$
\begin{array}{r}
K_{i \nu}(\nu z)=\frac{\pi 2^{1 / 2}}{\nu^{1 / 3}} \exp \left(-\frac{\pi}{2} \nu\right)\left(\frac{\zeta}{z^{2}-1}\right)^{1 / 4}\left\{A i(\xi)\left[1+\sum_{s=1}^{m} \frac{A_{s}(\zeta)}{\nu^{2 s}}\right]\right. \\
\left.+\frac{A i^{\prime}(\xi)}{\nu^{4 / 3}} \sum_{s=0}^{m-1} \frac{B_{s}(\zeta)}{\nu^{2 s}}+\frac{\exp \left\{-\frac{2}{3} \xi^{3 / 2}\right\}}{1+|\xi|^{1 / 4}} \cdot O\left(\nu^{-2 m-1}\right)\right\},
\end{array}
$$

where $\xi=\nu^{2 / 3} \zeta, \frac{2}{3} \zeta^{3 / 2}=\left(z^{2}-1\right)^{1 / 2}-\operatorname{arcsec} z$, and the coefficients are given by

$$
A_{s}(\zeta)=\sum_{m=0}^{2 s}(-1)^{m} b_{m} \zeta^{-3 m / 2} U_{2 s-m}, \zeta^{1 / 2} B_{s}(\zeta)=\sum_{m=0}^{2 s+1}(-1)^{m} a_{m} \zeta^{-3 m / 2} U_{2 s-m+1},
$$

with

1 The material of this note is contained in the author's doctoral dissertation at Oregon State University. The author wishes to thank Professor F. Oberhettinger for the suggestion of the problem and for his helpful criticism. 
and

$$
\begin{aligned}
& a_{0}=1, \quad a_{s}=\frac{(2 s+1)(2 s+3) \cdots(6 s-1)}{s !(144)^{*}}, \\
& b_{0}=1, \quad b_{s}=-\frac{6 s+1}{6 s-1} a_{s}
\end{aligned}
$$

$$
\begin{aligned}
& U_{0}=1, \\
& U_{1}=\frac{1}{8} v\left[\frac{5}{3} v^{2}+1\right], \\
& U_{2}=v^{2}\left[\frac{9}{128}+\frac{77}{192} v^{2}+\frac{385}{1152} v^{4}\right], \\
& U_{3}=v^{3}\left[\frac{75}{1024}+\frac{4563}{5120} v^{2}+\frac{17017}{9216} v^{4}+\frac{85085}{82944} v^{6}\right], \cdots,
\end{aligned}
$$

where $v=1 /\left(z^{2}-1\right)^{1 / 2}$.

As a consequence of the uniformity of (2) we obtain the Hankel $[1$, p. 23] and the Debye-type series for $z=\nu / p$ with $p>1$ and $p<1$, respectively (which can be derived by the method of steepest descent) by substituting the appropriate asymptotic expansions of the Airy function $[5,(\mathrm{~A} 6)$ and (A7)] in (2). If $p \sim 1$, the Debye-type series fail to give satisfactory approximation; for $|z-\nu|=O\left(z^{1 / 3}\right), \operatorname{Re} z>0$ an asymptotic expansion can be given in terms of the Airy function using Schoebe's method [7], this series also can be deduced from (2) as a special case.

The uniform asymptotic expansion of

$$
K_{i \nu}^{\prime}(\nu z) \equiv \frac{d}{d(\nu z)} K_{i \nu}(\nu z)
$$

is derived using Olver's Theorem B. We have, for $z \in R$ and $\nu \rightarrow \infty$,

$$
\begin{aligned}
K_{i \nu}^{\prime}(\nu z) \sim & \frac{2^{1 / 2} \pi e^{-\pi \nu / 2}}{z}\left(\frac{\zeta}{z^{2}-1}\right)^{-1 / 4} \\
& \cdot\left\{\frac{A i(\xi)}{\nu^{4 / 3}} \sum_{s=0}^{\infty} \frac{C_{s}(\zeta)}{\nu^{2 s}}+\frac{A i^{\prime}(\xi)}{\nu^{2 / 3}} \sum_{s=0}^{\infty} \frac{D_{s}(\zeta)}{\nu^{2 s}}\right\},
\end{aligned}
$$

where

$$
\begin{aligned}
& D_{0}=1, \quad C_{0}=\zeta^{1 / 2} V_{1}+\frac{7}{48} \zeta^{-1}, \\
& D_{1}=\frac{455}{4608} \zeta^{-3}-\frac{5}{48} \zeta^{2} C_{0}+V_{2}, \cdots,
\end{aligned}
$$


and

$$
V_{1}=-\left[\frac{3}{8}+\frac{7}{24} v^{2}\right] v, \quad V_{2}=\left[\frac{15}{128}+\frac{33}{64} v^{2}+\frac{455}{1152} v^{4}\right] v^{2}, \cdots
$$

Again this series gives the Debye-type series of $K_{t \nu}^{\prime}(\nu z)$ as special cases.

We note that the first terms in (2) and in (3) for real values of $z$ give the corresponding asymptotic formulae derived in [2].

Since $A i(z)$ and $A i^{\prime}(z)$ have only real negative zeros it can be deduced from (2) and (3) that all zeros of $K_{i \nu}(\nu z)$ and of $K_{i \nu}^{\prime}(\nu z)$ lie asymptotically on the segment $-1<z<1$ of the real axis.

By inverting (2) and (3) asymptotic expansions for the sth zero $k_{\nu, s}$ of $K_{i \nu}(z)$ and for the sth zero $k_{\nu, s}^{\prime}$ of $K_{i \nu}^{\prime}(z)$ are obtained.

$$
k_{\nu, 8} \sim \nu \sum_{r=0}^{\infty} \frac{p_{r}(\alpha)}{\nu^{2 r}}
$$

where

$$
p_{0}=z, \quad p_{1}=\alpha_{1} z^{\prime}, \quad p_{2}=\alpha_{2} z^{\prime}+\frac{1}{2} \alpha_{1}^{2} z^{\prime \prime}, \cdots
$$

and

$$
\alpha_{1}=-B_{0}, \quad \alpha_{2}=-\left[B_{1}+\alpha_{1} B_{1}^{1}+\frac{1}{2} \alpha_{1}^{2} B_{0}^{2}+\frac{1}{6} \alpha_{1}^{3} B_{0}^{8}\right]
$$

with

$$
\begin{aligned}
& B_{0}^{1}=1, \\
& A_{r}^{2 m}=\frac{d}{d \zeta} A_{r-1}^{2 m-1}+\zeta B_{r}^{2 m-1}, \quad A_{r}^{2 m+1}=\frac{d}{d \zeta} A_{r}^{2 m}+\zeta B_{r}^{2 m}, \\
& B_{r}^{2 m}=A_{r}^{2 m-1}+\frac{d}{d \zeta} B_{r}^{2 m-1}, \quad B_{r}^{2 m+1}=A_{r}^{2 m}+\frac{d}{d \zeta} B_{r-1 .}^{2 m} . \\
& k_{\nu, s}^{\prime} \sim \nu \sum_{r=0}^{\infty} \frac{g_{r}(\beta)}{\nu^{2 r}},
\end{aligned}
$$

where the coefficients $g_{r}(\beta)$ are given by (5) with the symbols $p_{r}$ and $\alpha_{r}$ are replaced by $g_{r}$ and $\beta_{r}$, respectively. In place of (6) we have

$$
\beta_{1}=-\zeta^{-1} C_{0}, \quad \beta_{2}=-\zeta^{-1}\left(C_{1}+\beta_{1} C_{1}^{1}+\frac{1}{2} \beta_{1}^{2} C_{0}^{2}+\frac{1}{6} \beta_{1}^{3} C_{0}^{3}\right),
$$


where

$$
\begin{array}{ll}
C_{r}^{2 m}=\frac{d}{d \zeta} C_{r}^{2 m-1}+\zeta D_{r}^{2 m-1}, & C_{r}^{2 m+1}=\frac{d}{d \zeta} C_{r-1}^{2 m}+\zeta D_{r}^{2 m}, \\
D_{r}^{2 m}=C_{r}^{2 m-1}+\frac{d}{d \zeta} D_{r-1}^{2 m-1}, & D_{r}^{2 m+1}=C_{r}^{2 m}+\frac{d}{d \zeta} D_{r}^{2 m} .
\end{array}
$$

These expansions are uniform with respect to the enumeration $s$ of the zeros and the error on terminating the expansions at the $r$ th term is $O\left(\nu^{-2 r-1}\right)$ and $O\left(\nu^{-2 r / 3-1 / 8}\right)$, respectively. For numerical calculations the coefficients $p_{r}(\zeta)$ and $g_{r}(\zeta)$ may be pretabulated.

\section{REFERENCES}

1. A. Erdélyi, Higher transcendental functions, Vol. 2, McGraw-Hill, New York, 1953.

2. F. G. Friedlander, Diffraction of pulses by a circular cylinder, Comm. Pure Appl. Math. 2 (1954), 705-732.

3. N. N. Lebedev, Sur une formule d'inversion, C. R. (Doklady) Acad. Sci. URSS (N. S.) 52 (1946), 655-658.

4. F. W. J. Olver, The asymptotic solutions of linear differential equations of the second order for large values of a parameter, Philos. Trans. Roy. Soc. London Ser. A. 247 (1954), 307-327.

5. - The asymptotic expansions of Bessel functions of large order, Philos. Trans. Roy. Soc. London Ser. A. 247 (1954), 328-368.

6. - The asymptotic solutions of linear differential equations of the second order in a domain containing one transition point, Philos. Trans. Roy. Soc. London Ser. A. 249 (1956), 65-97.

7. W. Schoebe, Eine an die Nicholsonformel anschliessende asymptotische Entwicklung fuer Zylinderfunktionen, Acta Math. 90 (1954), 265-307.

Portland State College 